\title{
[gw22-e0428] ANALYSIS OF LEFT VENTRICULAR REMODELLING IN PATIENTS TREATED WITH CARDIAC RESYNCHRONISATION THERAPY
}

Zhe An, Wenqi Zhang China-Japan Union Hospital of Jilin University

10.1136/heartjnl-2011-300867.528

Objective Matrix metalloproteinases (MMPs) and their tissue inhibitors (TIMPs) play a role in left ventricular structural remodelling. The aim of our study was to analyse MMP-2 and TIMP-1 levels as predictors of poor response to cardiac resynchronisation therapy (CRT).

Methods A 1-year cohort of patients who received a CRT device in our centre was included in the study. All patients underwent a 12-lead ECG, echocardiogram and clinical evaluation prior to implant and at 6- and 12-month follow-up. At the time of CRT implant, blood samples from a peripheral vein and the CS were obtained simultaneously. Serum and plasma samples were stored at $-80^{\circ} \mathrm{C}$ until assay. Serum MMP-2 and plasma TIMP-1 levels were assessed using a commercially available sandwich enzyme immunoassay.

Results A cohort of 84 CRT patients from our centre was prospectively evaluated at baseline and after 12-month follow-up. MMP-2 and TIMP-1 assays were performed prior to CRT implant. Cardiac resynchronisation therapy responders were defined as patients who survived, not transplanted, and increased their basal 6 min walking distance test (6MWDT) by $\geq 10 \%$ or improved their NYHA functional class. Over all, 50 patients $(60 \%)$ were classed as responders. At 12 -month follow-up, 12 patients (14.2\%) had died and 2 (2.4\%) patient had been transplanted. Compared with responders, non-responders had higher levels of TIMP-1 (277 \pm 59 vs $216 \pm 46 \mathrm{ng} /$ $\mathrm{ml}, \mathrm{p}=0.001)$, MMP-2 (325 \pm 115 vs $258 \pm 56 \mathrm{ng} / \mathrm{ml}, \mathrm{p}=0.02)$. In a multivariate analysis, TIMP-1 was the predictor of non-response to CRT (OR 0.97, 95\% CI 0.96 to 0.99, p=0.005).

Conclusion TIMP-1 was an predictor of non-response in patients treated with CRT. TIMP-1 and MMP-2 were higher in patients who did not respond to CRT. 